\title{
COLLABORATIVE WORK TOOLS IN LEARNING ENVIRONMENTS
}

Application in the University of Las Palmas

\author{
Mario Marrero ${ }^{1}$, Celso Perdomo ${ }^{1}$, Jorge Rodríguez ${ }^{2}$ and Antonio González ${ }^{2}$ \\ ${ }^{1}$ Cabildo de Gran Canaria, C/ Tomás Morales, 35002, Las Palmas GC, Spain \\ ${ }^{2}$ University of Las Palmas de Gran Canaria, Edificio de Ciencias Básicas, Campus \\ Universitario de Tafira, 35017, Las Palmas GC. Spain
}

\begin{abstract}
In this paper, we present HTC, an Internet package for collaborative work. This package is a collaborative and distributed application, which allows users to create their own individual workspaces, which, can in turn, be shared partially or totally with other HTC users. HTC fulfils the following basic objectives: intuitive and easy to use, easy to incorporate into an organization, and allows for global access to resources. Additionally, we present the tool's integration process into the University of Las Palmas de Gran Canaria. From this integration process and the statistical results, we obtain some conclusions. HTC is in its evaluation phase in various Spanish-speaking universities and companies.
\end{abstract}

Key words: CSCW (Computer Supported Cooperative Work), CSCL (Computer Supported Collaborative Learning, Groupware)

\section{INTRODUCTION}

With the implementation of information technologies in organizations, research in the cognitive field was initially focused on the study of the interaction between a human and a machine. In other words, the relationship that exists between an individual user and a computer system for the completion of a task.

This initial approach omits the collaborative aspect that many tasks have. Work is a social phenomenon. There exist thousands of connections and

The original version of this chapter was revised: The copyright line was incorrect. This has been corrected. The Erratum to this chapter is available at DOI: 10.1007/978-0-387-35689-1_19 
relationships among all of the parties involved in any production process. In general, the most interesting part of any task that provides the highest productivity is that groups work to achieve one common objective. Some statistics show that office workers spend between $30 \%$ and $70 \%$ of their time in meetings (Cole 1995). For example, in any company there are groups of people dedicated to corporate planning, budgeting, production, sales and other tasks. Nonetheless, many of the most extensive software applications available on the market at this time tend to ignore this fact, providing only for individual work environments.

Collaborative work is necessary due to the following factors: a) Problems are more and more complex, b) Problems are bigger, c) Problems are more and more specialized and d) The solutions to these problems require different aptitudes or abilities.

As a consequence, this brings about a change in attitude, since a) people need to work as members of a team (collaboration); b) people need to exchange information frequently and c) the success of the task not only depends on the merits of each individual worker, but also on the level of cooperation. On the other hand, it implies an organizational change due to the organization's own dynamic bringing about new forms (for permanent adaptation).

On the other hand, it is true that as collaborative work becomes more and more a part of an organization, all of the information that is generated can be filtered, analysed and organized generating knowledge.

In reality, the challenge for many organizations (Dix 1996) consists of being able to use tools such as HTC to eventually know what the organization already knows and have this information be accessible by any team member (with the appropriate permission rights) and from any location within the organization. With HTC, any organization can begin to develop a collaborative project and manage information starting with the following three fundamental objectives:

- Constantly learn how to improve and increase output from meetings and work groups within the organization.

- Interrelate the various work groups and the information they generate.

- Consolidate information to reach a higher level of learning.

The University of Las Palmas de Gran Canaria (UPLGC) has opted to answer these questions generating a system of layers, starting with the physical infrastructure, passing through the Online Information Service UPLGC, the work in groups and finishing off with information management.

This work is presented in five sections. In section 2, we discuss groupware technologies and the basic concepts known as 3Cs. In section 3, we introduce the collaborative work tool design and some aspects of utilization. Followed by the integration process of the tool in the University 
of Las Palmas de Gran Canaria, and finally, we present some conclusions and future work.

\section{GROUPWARE TECHNOLOGIES (WORK IN GROUPS)}

We can define a collaborative application as one that helps in the coordination of members of a group with the objective to achieve a common task. In general, this is a distributed application, such that, each participating member of the group works from his/her own workstation, sharing information with the rest of the members of the group.

Additionally, groupware is defined as a technology that allows groups of people to work in teams. The main objective of these technologies is to improve the productivity of work groups. Groupware is supported by three basic pillars - the 3Cs (Becker and Zanella 1998):

1. Communication. The members of a work group need to communicate with each other to exchange information.

2. Collaboration. Members of the group collaborate contributing, submitting feedback, combining opinions and generating documentation together through the exchange of information.

3. Coordination. Groupware technologies allow for the coordination of different members of a group in order to carry out a particular project in common.

\section{HTC UTILIZATION}

HTC is a collaborative and distributed application where you can create an individual workspace for each user, which can in turn, be shared partially or totally with other HTC users. Sharing workspace area is made possible by establishing collaborative relationships among HTC users.

Below is a list of fundamental requirements needed of HTC within an organization:

- Ease of use. The tool should be easy to use by any person with limited knowledge of information technology.

- Global access. The tool should do away with spatial barriers that may exist between various members of a workgroup.

- Easy to incorporate into an organization. The integration of HTC into any organization should not involve traumatic software migrations to the new system. 
- Access to centralized resources. Easily create a repository of information resources available in the tool.

The tool for collaborative work, HTC, offers the following functionality:

- Structure based on objects. HTC simplifies its internal structure into three fundamental objects: folders, files, and notes. Based on these three main objects you can create more complex objects such as discussion forums or work flow systems.

- Internal events component. The basic events defined in HTC are: reading an object, modifying an object, and writing to an object.

- Security component. Authentication by means of a username and password (or optionally the incoming IP address). Access rights on folders.

- Distinct work areas. Users access their personal workspace when entering the HTC tool. All folders, files, and notes in this workspace belong to the user, and by default, cannot be accessed by any other user. To share any of these objects with another member, the user will need to create a Shared Workspace. This is accomplished by creating a folder, and assigning read/write permissions to the users who will access the shared folder, and will form part of a workgroup.

- Discussion and debate forums component. This is possible as a result of the implementation of the notes object.

- Auditing and Sorting component.

- Work with 124 different file formats. Among the most common files are Word (doc), Adobe Acrobat (pdf), and Powerpoint (ppt). Potentially upload and download files quickly. Recyclable wastebasket.

- Address book. Contains contact information of other HTC users who will participate in your projects.

- Invite other colleagues to participate in a workgroup.

- Create accounts instantly. Without e-mails, without delays, without waiting to receive a confirmation or depending on technical staff to create the account for you.

- Intuitive and easy-to-use web interface.

- Administrator component. Access databases containing registered user information, auditing information, suggestions (based on design or content), internal access statistics, etc.

\subsection{Connecting to $\mathrm{HTC}$ and registering}

HTC is an Internet-based tool which can only be accessed if a user has an Internet connection and browser (IE or Netscape). The URL of the HTC tool is http://www.ulpgc.es/htc. The home page is shown in Figure 11-1. 
In this figure, you will find a "New user" icon. Click on it to access the registration form. Choose a username and password with which you will authenticate your identity in HTC. Completing the form automatically creates an account for you or a colleague quickly, easily and immediately.

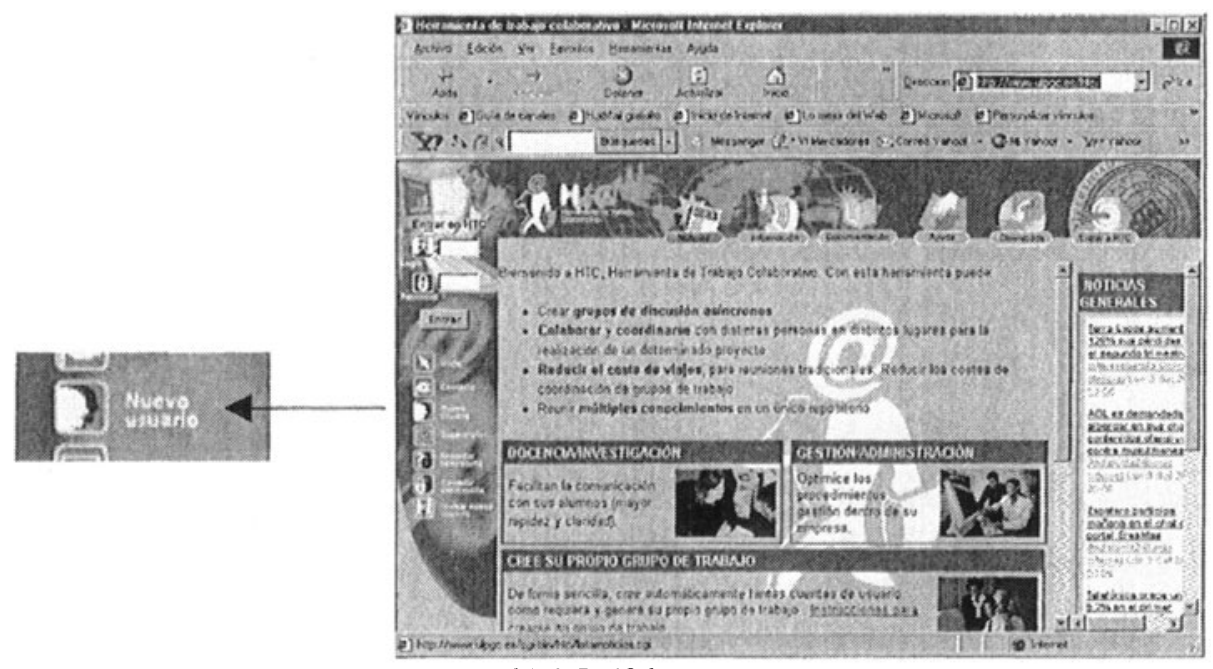

Figure 11-1. HTC home page.

Once the account has been created, access your personal workspace by entering your user name and password into the textbox located on the upper left-hand corner of the home page (Figure 11-1).

\subsection{The personal workspace}

In your private workspace, you can organize all of your information in folders, files and notes. HTC allows you to share all or part of your personal work area with other HTC members.

The workspace is divided into 2 different areas: chief icons and the work area. The chief icons are arranged according to the three types of basic objects, which are part of the HTC tool:

- Folders. Allow you to organize projects and workgroups which you are involved in.

- Files. Any type of file in any format which contains information (Word documents, Reports, Articles, Images, Sound files, Video files, etc.).

- Notes. Information stored as plain text containing feedback: opinions, comments, objections, etc. Create discussion forums with the notes object.

There are six chief icons: New, Edit, Delete, Cut, Copy and Paste. Figure 11-2 shows the tool's chief icons. 


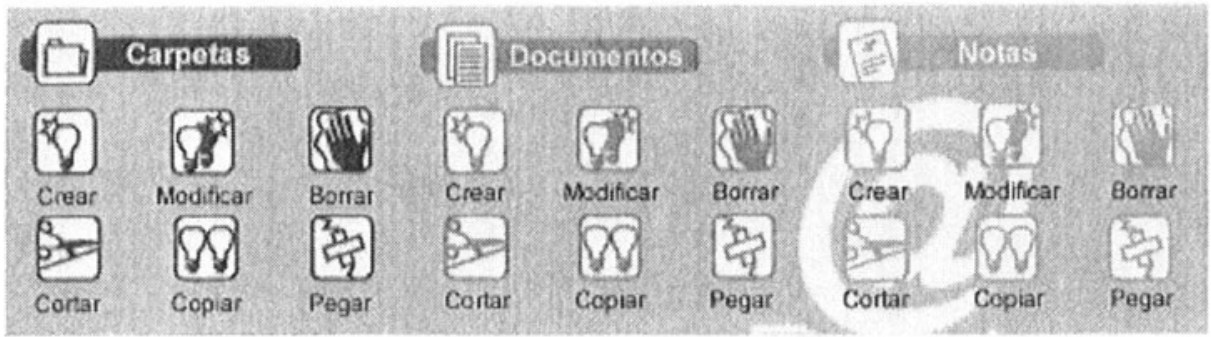

Figure 11-2. Tool bar with chief icons for each type of object: folders, files, and notes.

\subsection{Creating folders}

Folders are objects that allow you to organize documents, discussion or debate forums, etc., based on the arrangement you choose for your workspace. For example, you could have a folder for each project you work on, or a folder for each file format you are using, etc.

For example, imagine you want to create a folder where you store the job proposals for the students in your class. Click on the new icon in the Folders section, type the name of the folder "Job Proposals", and click on the Submit button. With these simple steps, you created a new folder similar to what is shown in Figure 11-3.

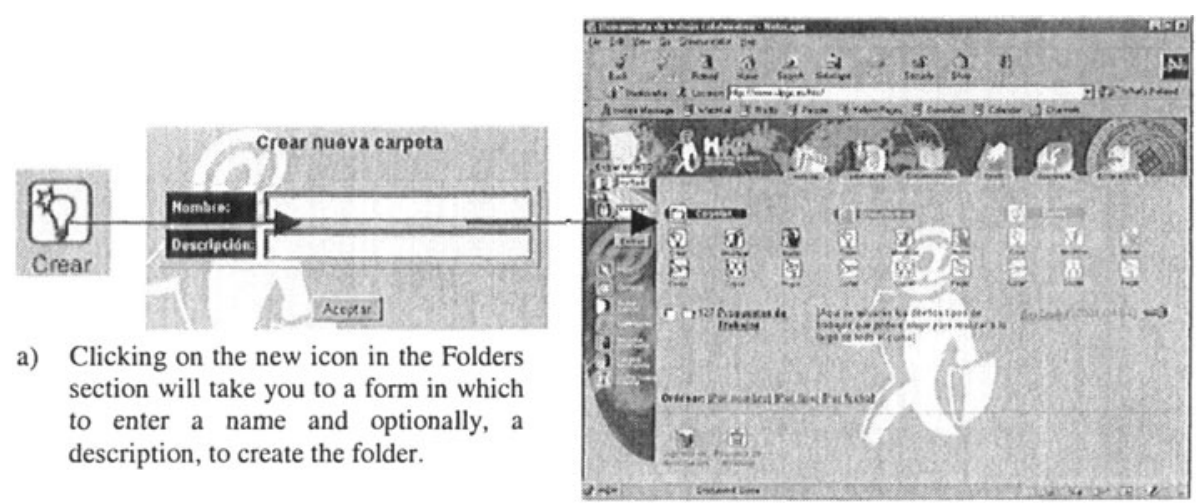

Figure 11-3. Sequence of steps to create a folder.

Additionally, you can modify the folder properties, cut or copy the folder to later paste in another location of our workspace. 


\subsection{Creating files}

Files are archives in any format. These can range from documents written in Word, presentations developed in PowerPoint, databases, spreadsheets, images, MP3 sound files, videos, etc.

To create a file, click on the new icon in the Files section. In the file creation form, insert the name of the new file, a description (optional), browse to your hard drive for the file to upload to HTC, and choose the appropriate file extension (doc for Word documents, ppt for PowerPoint presentations, etc.).

\subsection{Creating notes}

Notes are plain text messages that allow you to communicate information quickly, and also establish discussion forums in HTC with other members. Creating notes is much easier than creating files (as specified in the previous section). Clicking on the new icon will take you to a form where you will write the text you want to include in the note.

\subsection{Address book}

In HTC, you can have an address book with your contacts: people with whom you collaborate on projects, clients, etc.

To add a new contact to your address book, click on the Address Book icon and add a new member. You should know the user name of the person you want to add to your address book, and of course, this person should have an open account at HTC.

\subsection{Sharing the workspace}

As mentioned earlier, members of HTC create their own accounts and access their own personal workspaces. In this workspace the users can organize and include as many documents as they want, keeping in mind that only they can see them.

If you want to share all or part of the information in your workspace with one or more users, then you will need to create a shared folder. The procedure to share a folder, and any information you store in it, is simple and easily accomplished following three easy steps defined in the online manual. 


\section{HTC APPLICATION IN A UNIVERSITY ENVIRONMENT. A CASE STUDY: ULPGC}

The ULPGC has at its disposal the Online Information Service (OIS) that fulfils two fundamental objectives: to be an open window to society and become a powerful nervous system for internal management.

The working philosophy that identifies OIS is based on the flow of information circulating directly from its origin towards its final destination on the Server, independent of the knowledge people that generate the information have of information technology.

To achieve this objective, on one hand, we have defined and created simple to use tools for any person foreign to the field of information technology, and on the other hand, the directors of the university have promoted and impelled the utilization of these tools among the university community.

In figure 11-4 we define the layers for the distinct technologies in the university. The hardware (HW) layer provides the physical resources, which permit the servers to function correctly (networks, mirrored systems, Unix technology, fault redundancy systems, etc.)

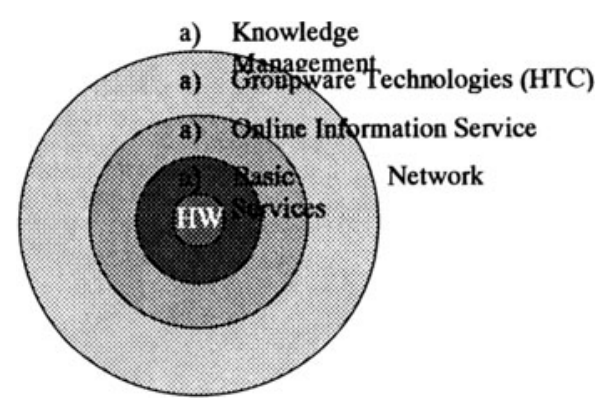

Figure 11-4. Structure of ULPGC's Information Service layers.

The layer, basic network service, provides the logical media, mainly, the communication protocols at the application level (http, dns, dhcp, etc.) Once the Online Information Service layer has been created, the university must approach the level of information management. To reach this goal, it becomes necessary to develop a culture on collaborative work within the community, where information is not published from just one location (or only one author), but that the information is a product of a group of people working together. Therefore, we add to the Online Information Service layer, 2 other layers: groupware and information management. 
The publication system in the groupware layer is based on different people working together to create one single document.

In its initial implementation phase, the HTC tool is being used in the University of Las Palmas de Gran Canaria to support and complement teachers and students in their interaction in some courses, among other uses. Thanks to HTC, professors can place didactic information (transparencies, previous exams, notes, etc.) at the student's disposal for a particular subject.

For these work groups in HTC we propose the following folder structure: Faculty, Academic Calendar, Course syllabus, Academic project, Academic resources, Grades, Bulletin boards and Suggestions box.

The tool is being used in courses such as Biomechanics Movement (Physical Education Department), Geodynamics (department of Marine Sciences), etc. After a month and a half, there are over 1877 registered users. The growth is exponential. At the time of writing, there are over 2441 documents, 1062 folders and 588 distributed notes available to the users.

\section{CONCLUSIONS AND FUTURE WORK}

From the utilization of the tool, we obtain the following conclusions:

- The group of students that use HTC use it as a repository for personal documents in their own private workspace. In this workspace, they place reports, course notes, experiments, programs, etc. They can access all of these resources independent of where they are, in a science lab or a computer lab, in their home or even in a cyber cafe.

- Professors use HTC as an additional channel through which they distribute additional documentation to the students. The confidentiality of the communication and interaction with the student is maintained since the students themselves define the model of collaboration and sharing of information.

- Debate forums are carried out with questions from the students. The answers are always visible to the students within this area. Course students can read every student's questions and answers. In this way we start to create an FAQ (Frequently Asked Questions) that can be used in future courses. Another advantage to this type of interaction is the anonymity where the user's fear to ask questions disappears.

- Each faculty member uses the HTC tool at their own rhythm. Given the intuitiveness of its usage, faculty staff generate new possibilities for its proper usage and incorporate them into their classes.

Future work includes in the first stage, to obtain experience in the management environment both in universities as well as other institutions 
(Cabildo de Gran Canaria and Cabildo de Fuerteventura, among others) since we suspect that the tool can reach its maximum potential in this field. Subsequently, based on this experience proceed with improving certain technical aspects, among them, the definition of a distributed architecture to support a higher number of users and the integration of mobile services for communication and administration. In addition, we are working in offering this package under GNU General Public License, to share it with other universities and educational institutions.

\section{REFERENCES}

Becker, K and Zanella A. L. (1998). A Cooperation Model for Teaching/Learning Modeling Disciplines. Instituto de Informática. Pontifícia Universidade Católica do Rio Grande do Sul. Porto Alegre. Brazil.

Cole P. (1995). The Impact of Group Context on Patterns of Groupware Use: A Study of Computer Conferencing as a Medium of Work Group Communication and Coordination. Center for Coordination Science. MIT (Massachusetts Institute of Technology).

Dix, A. (1996). Challenges and Perspectives for Cooperative Work on the Web. HCI

Research Centre, School of Computing and Mathematics. The University of Huddersfield.

UK. 
SECTION 4

ICT TRAINING FOR EDUCATIONAL PROFESSIONALS 